\title{
LEVANTAMENTO DE SUBSÍDIOS PARA ASSISTÊNCIA DE ENFERMAGEM AMBULATORIAL A PACIENTES PORTADORES DE VALVOPATIA AÓRTICA
}

\author{
Karin Mitie Kubo ${ }^{1}$ \\ Maria Cecilia Bueno Jayme Gallani \\ Rachel Noronha ${ }^{3}$ \\ Roberta Cunha Rodrigues Colombo ${ }^{4}$
}

Kubo KM, Gallani MCBJ, Noronha R, Colombo RCR. Levantamento de subsídios para assistência de enfermagem ambulatorial a pacientes portadores de valvopatia aórtica. Rev. Latino-am Enfermagem 2001 setembro-outubro; 9(5):55-62.

Este trabalho objetivou caracterizar o perfil do paciente ambulatorial com valvopatia aórtica, identificar o grau de conhecimento sobre sua doença, suas principais limitações no cotidiano e como ocorre o processo de enfrentamento nessa situação. Os dados obtidos através de entrevista semi-estruturada aplicada a 12 pacientes e analisados através de metodologia de análise quantitativa e qualitativa, permitiram identificar como estes sujeitos representam para si a doença e o tratamento, bem como suas implicações no cotidiano, ou seja, possibilitaram captar a realidade a partir da perspectiva do sujeito, um conhecimento imprescindivel para a elaboração de uma proposta educacional.

PALAVRAS CHAVE: educação do paciente, valva aórtica, cuidados de enfermagem

\section{SUBSIDIES TO ELABORATE OUTPATIENT NURSING CARE FOR PATIENTS WITH AORTIC VALVE DYSFUNCTION}

This study aimed at characterizing the profile of outpatients with aortic valve dysfunction, identifying their knowledge about the disease, their major limitations during everyday activities and their means of coping with their predicament. The data obtained by semistructured interviews with 12 patients and analyzed by quantitative and qualitative methods enabled to identify how the patients perceive the illness and the treatment as well as the implications to their everyday activities, that is, they allowed to capture reality from the subject's perspective, which is the knowledge for the elaboration of an educational proposal.

KEY WORDS: patient education, aortic valve, nursing care

\section{LEVANTAMIENTO DE SUBSIDIOS PARA LA ATENCIÓN DE ENFERMERÍA AMBULATORIA A PACIENTES CON VALVULOPATÍA AÓRTICA}

El objetivo del este trabajo, es caracterizar el perfil del paciente ambulatorio con valvulopatía aórtica, identificar su conocimiento sobre la enfermedad, las principales limitaciones en su cotidiano y como ocurre el proceso de enfrentamiento en ésta situación. Los datos obtenidos a través de entrevista semi-estructurada de 12 pacientes y analizados de acuerdo con la metodología de análisis cuantitativa y cualitativa, permitieron identificar como estos sujetos representan para si la enfermedad y su tratamiento, así como también sus implicaciones en el cotidiano, o sea, hicieron posible captar la realidad vista desde la perspectiva del sujeto, conocimiento imprescindible para la elaboración de una propuesta educativa.

PALABRAS CLAVES: educación para la salud, válvula aórtica, cuidados de enfermería

\footnotetext{
${ }^{1}$ Enfermeira do Instituto do Coração da Faculdade de Medicina da Universidade de São Paulo. ${ }^{2}$ Enfermeira, Doutor em Enfermagem, Professor Colaborador, e-mail: mcbjgallani@neuro.med.br; ${ }^{3}$ Enfermeira, Doutor em Educação, Professor Assistente; ${ }^{4}$ Enfermeira, Doutor em Enfermagem, Professor Colaborador, email: rcolombo@dglnet.com.br. Departamento de Enfermagem da Faculdade de Ciências Médicas da Universidade Estadual de Campinas
} 


\section{INTRODUÇÃO}

A convivência com os pacientes cardíacos e dentre eles os valvopatas tem nos despertado para a importância de elaborarmos um plano assistencial de enfermagem específico, principalmente para aqueles pacientes que encontram-se com a manifestação dos sinais e sintomas da afeç̧ão valvar e aguardam pela realização do procedimento intervencionista e sobretudo aqueles que mesmo após o procedimento cirúrgico, ainda apresentam reincidência dos sinais e sintomas. Estes pacientes convivem com sua sintomatologia mais ou menos exacerbada, de acordo com seu estado clínico e emocional, fortemente determinados por sua história clínica e de vida.

A manifestação clínica da valvopatia leva o indivíduo a experimentar limitações fisiológicas, sociais e emocionais, que determinarão mudanças no seu estilo de vida, relacionadas, principalmente às atividades de vida diárias. Tais modificações parecem interferir de modo contundente na qualidade de vida desses indivíduos ${ }^{(1)}$

Cada vez mais, tem sido expressivo o número de publicações direcionados à compreensão e mensuração de qualidade de vida, com contribuição de diferentes áreas como psicologia, enfermagem, sociologia, medicina, economia e antropologia. Essa diversidade tem implicado em diferentes perspectivas de abordagem da questão, e conseqüentemente na falta de um consenso sobre a definição de seu conceito e, portanto, de como mensurá-lo( ${ }^{(2)}$. A definição de qualidade de vida como "o grau de satisfação do indivíduo com a sua vida e o grau de controle que exerce sobre ela"(3) e qualidade de vida ligada à saúde, como "o grau de limitação e desconforto que a doença e/ou sua terapêutica acarretam ao paciente e sua vida"(3), entretanto, podem ser utilizadas para apontar algumas das características marcantes do que seria qualidade de vida. Ou seja, a qualidade de vida pode ser entendida em termos das expectativas pessoais do paciente e se foram ou não atingidas ou alcançadas ${ }^{(4)}$.

De posse dessa compreensão o profissional deve ter presente, em seu processo de decisão, que a compreensão e a avaliação correta dos dados subjetivos do paciente são seus aliados para que $\mathrm{o}$ indivíduo possa ser conduzido à adesão ao tratamento $\mathrm{e}$ torne-se participante ativo do processo de mudança ${ }^{(4)}$.

Sendo assim, ao analisar-se a qualidade de vida de um paciente, de acordo com uma concepção mais atualizada da prática de saúde, é indispensável considerar-se tanto aspectos objetivos como subjetivos, ou seja, as informações objetivas, tradicionais, baseadas em indicadores biomédicos e as subjetivas, derivadas dos valores e crenças do próprio paciente ${ }^{(4)}$.

Além da consideração dos aspectos psicossociais do sujeito alvo das intervenções, é imprescindível a inclusão da compreensão também da família, do modo como eles (paciente-família) experienciam a doença e como têm conduzido sua vida ${ }^{(5)}$.

As publicações atuais, em maior número, têm sido direcionadas à fisiopatologia das valvopatias, ou relacionadas ao período trans ou pós-procedimento terapêutico - valvoplastia ou cirurgia, apesar da importância das intervenções que auxiliam 0 indivíduo a viver da melhor maneira. Ainda são poucos os estudos, que direcionam a assistência de enfermagem no período clínico, no qual o paciente já experimenta sinais e sintomas por vezes, muito significativos, porém ainda não se encontra na fase de indicação de cirurgia.

Tal preocupação levou-nos a desenvolver um trabalho de pesquisa junto a um grupo específico de pacientes valvopatas para avaliar como a doença poderia estar interferindo em sua qualidade de vida. Optamos por iniciar o trabalho junto aos pacientes com valvopatia mitral, visto que nestes resume-se um determinado grupo de manifestações hemodinâmicas e clínicas peculiares, e conseqüentemente com demanda de condutas específicas ${ }^{(6)}$. Este estudo mostrou-nos que as repercussões hemodinâmicas e clínicas da valvopatia mitral determinam modificações no estilo de vida do indivíduo, o qual carece de apoio necessário para adaptar-se adequadamente a tais mudanças. Sendo assim, sentimo-nos estimuladas a dar continuidade ao estudo, desta vez com os pacientes portadores de valvopatia aórtica (estenose/insuficiência) que reúnem sinais/sintomas tão diferentes do paciente com valvopatia mitral, quanto são diferentes suas repercussões hemodinâmicas e que, portanto, necessitam de um programa de educação em saúde que atenda às questões que são comuns aos pacientes valvopatas, mas também às especificidades de sua patologia.

Assim, este estudo tem como objetivo: caracterizar o perfil do paciente ambulatorial, com disfunção valvar aórtica (estenose e insuficiência); identificar o grau de conhecimento do valvopata sobre a doença; identificar as principais limitações no cotidiano e como ocorre o processo de enfrentamento nessa situação; e subsidiar a elaboração de um plano assistencial em enfermagem.

\section{SUJEITOS E MÉTODOS}

A população deste estudo foi constituída por 12 pacientes portadores de estenose e/ou insuficiência aórtica, atendidos no Ambulatório de Cardiologia do Hospital de Clínicas da Universidade Estadual de Campinas (UNICAMP), no período de novembro de 1998 a fevereiro de 1999. Todos comunicavam-se verbalmente, maiores de dezoito anos e concordaram em participar da pesquisa. Os dados foram obtidos através de entrevista semi-estruturada com o paciente e consulta ao prontuário hospitalar para levantamento de dados 
pertinentes. As entrevistas foram gravadas em fita cassete e posteriormente transcritas com a finalidade de obter-se integralmente o discurso dos pacientes entrevistados. 0 estudo obteve a aprovação do Comitê de Ética em Pesquisa da Faculdade de Ciências Médicas - UNICAMP e foi garantido aos pacientes o sigilo e 0 anonimato, assegurado pelo consentimento informado assinado pelos sujeitos da pesquisa.

O roteiro de entrevista foi validado através de um estudo piloto envolvendo cinco pacientes. Os itens fechados foram analisados de modo quantitativo e seus resultados expressos em números absolutos e percentuais; as questões abertas foram analisadas de modo qualitativo. Esta análise teve como base a realização de leitura e re-leitura cuidadosas de cada discurso, seguida da leitura transversal da mesma questão de todos os entrevistados. Foram identificados núcleos comuns - latentes ou manifestos - nos discursos, que após terem sido codificados, convergiram para o que chamamos de categoria temática. Destaca-se que para esta análise, procurou-se adotar medidas que garantissem a qualidade e validade da obtenção e análise dos dados, como preparo do entrevistador para garantir sua credibilidade junto ao grupo entrevistado, bem como a adoção de um método de análise analiticamente rigoroso, mentalmente replicável e explicitamente sistemático ${ }^{(7)}$.

\section{RESULTADOS E DISCUSSÃO}

A população estudada, foi constituída em sua maioria (83\%) pelo sexo masculino com idade entre 34 e 78 anos, concentrando-se na faixa dos 30-44 anos. Quanto à procedência, seis pacientes eram da Região Administrativa de Campinas ${ }^{*}$, cinco de Campinas e apenas um externo a esta área. A maioria dos pacientes $(75 \%)$ possuía 0 primeiro grau incompleto, $17 \%$ eram analfabetos e apenas $8 \%$ possuíam o primeiro grau completo. Quanto à ocupação, oito $(66,7 \%)$ pacientes referiram manter a atividade de trabalho. Já quatro $(33,3 \%)$ restantes deixaram de exercer suas respectivas funções, aposentando-se por invalidez ou requerendo afastamento de suas funções. Quanto à renda familiar, houve uma concentração na faixa de quatro a seis salários mínimos $(58,3 \%)$.

Quanto ao diagnóstico médico da doença valvar, encontramos que cinco pacientes $(41,7 \%)$ possuíam dupla lesão (estenose e insuficiência aórtica), encontrando o mesmo valor para a insuficiência aórtica; dois pacientes (16,7\%) apresentavam estenose aórtica. Vale salientar o fato de que a hipótese diagnóstica acima descrita refere-se à patologia cardíaca predominante, sendo possível a presença de outro tipo de patologia associada. Sabe-se, que de acordo com a idade em que a valvopatia é detectada, pode-se inferir o tipo de afecção que levou à degeneração valvar ${ }^{(5)}$. Assim, abaixo dos 30 anos, é muito provável que a causa seja congênita; na faixa etária de 30-70 anos, a valvopatia é provavelmente devido à doença cardíaca reumática; e acima dos 70 anos de idade, a causa mais freqüente é a calcificação dos folhetos valvares. Dos pacientes estudados, foi possível encontrar o registro da etiologia em apenas sete dos 12 prontuários levantados. Destes, seis apontavam a febre reumática e um relatava endocardite bacteriana como etiologia.

Quanto ao tipo de tratamento médico realizado, sete pacientes (58,3\%) encontravam-se em tratamento clínico, sem nunca ter realizado algum tipo de tratamento cirúrgico e cinco $(41,7 \%)$ já haviam sido submetidos à troca valvar, sendo quatro com colocação de prótese biológica e um com prótese metálica.

De todos os pacientes entrevistados, apenas um referiu nenhum conhecimento sobre sua doença. Os demais (11/12) emitiram representações que têm sobre a doença: "válvula não funcionante", "repercussão da doença na vida do sujeito", "relação da doença com necessidade de intervenção" e "construção imprecisa da doença", conforme evidencia a Tabela 1. Ainda em relação a esta questão, consideramos ser importante fazer o destaque de alguns dados, tais como 0 fato de mais da metade dos pacientes (7/12) fazerem referência ao termo "entupimento da válvula", mesmo que o problema médico fosse a insuficiência aórtica isoladamente (presente em cinco dos 12 pacientes). Dentro deste mesmo grupo, chamou-nos a atenção a fala de um paciente (1/7), que mesmo após realizar a cirurgia de troca valvar, não reconhecia a mesma como problema de saúde. Na categoria "relação da doença com necessidade de intervenção", verificou-se que dos seis pacientes (6/12) que citaram esta categoria, quatro (4/6) não tinham sido ainda submetidos a uma cirurgia cardíaca. Outros quatro (4/12) fizeram referência à categoria "repercussão da doença na vida do sujeito" e três (3/12) relatos de pacientes foram incluídos na categoria "construção imprecisa do conceito da doença", pelo fato de citarem vários elementos, sem no entanto relacioná-los, dentro do que chamaríamos de lógica para construção de um conceito.

Tabela 1 - Distribuição da freqüência e porcentagem das respostas dos pacientes $(n=12)$, quanto ao conhecimento sobre sua doença, segundo as categorias temáticas, Campinas, 1999

\begin{tabular}{|c|c|c|}
\hline CATEGCRIASTEMḾTICAS & $\mathrm{N} \neq$ & of \\
\hline 'Vákula não funcionante & 7 & 583 \\
\hline Construção imprecisa do conceito da doença & 3 & 25,0 \\
\hline Repercussão da doençana vida do sujeito & 4 & 33,3 \\
\hline Relação da doença com necessidade de intervenção & 6 & 500 \\
\hline
\end{tabular}

* Cada um dos pacientes pode ter apontado mais de uma categoria

* Americana, Artur Nogueira, Cosmópolis, Engenheiro Coelho, Estiva Gerbi, Holambra, Hortolândia, Indaiatuba, Itapira, Jaguariuna, Mogi Guaçu, Mogi Mirim, Monte Mor, Nova Odessa, Paulínia, Pedreira, Santa Bárbara D’Oeste, Santo Antonio de Posse, Sumaré, Valinhos, Vinhedo ${ }^{(8)}$ 
Quando questionados sobre qual a causa do problema, dos 12 pacientes entrevistados, quatro relataram a etiologia específica da doença; três pacientes desconheciam a causa e cinco atribuíramna a fatores que, de acordo com a literatura, não guardam relação com a etiologia do problema da válvula aórtica.

De acordo com a literatura os principais tipos de estenose aórtica adquirida podem ser de origem reumática; de envolvimento reumatóide; degenerativa senil calcificada e aterosclerótica ${ }^{(9)}$. Já a insuficiência aórtica, pode ser causada por doença primária ou dos folhetos da válvula aórtica ou da parede da raiz aórtica ou de ambos ${ }^{(10)}$. Outras causas valvares primárias de insuficiência aórtica incluem a endocardite infecciosa, a dilatação acentuada da aorta ascendente e a febre reumática, sendo esta última a causa mais freqüente de doença valvar primária causando insuficiência. Destacase, que a cardiopatia de origem reumática continua sendo um problema de saúde comum nos países em desenvolvimento, determinando morbidade e mortalidade, tanto em crianças como em adultos $^{(11)}$.

Ao indagarmos sobre as orientações recebidas sobre a doença, 10 (10/12) pacientes informaram ter recebido orientações específicas, que estão ilustradas na Tabela 2. Dentre as orientações referidas, destaca-se a categoria: "Cuidados decorrentes da existência da doença", sendo que o profissional que forneceu estas orientações para a maioria dos pacientes foi o médico $(9 / 10)$. Estes resultados assemelham-se aos achados do estudo com portadores de valvopatia mitral ${ }^{(6)}$, em que os autores verificaram que a maioria dos pacientes que receberam informações caracterizavam-nas como relativas aos cuidados decorrentes da existência da doença, além de todas as orientações terem sido fornecidas pelo médico.

Tabela 2 - Distribuição da freqüência e porcentagem das respostas dos pacientes $(n=10)$ que receberam orientações sobre sua doença, segundo as categorias temáticas, Campinas, 1999

\begin{tabular}{|c|c|c|}
\hline CRTEGCRIAS TEMḾTIOAS & $N^{p *}$ & ${ }_{\%}^{\circ}$ \\
\hline Manutenção do estilo de vida & 1 & 10 \\
\hline Importância da adesão à conduta terapêuttica & 4 & 40 \\
\hline Cuidados decomentes da existência da doença & 7 & 70 \\
\hline
\end{tabular}

* Cada um dos pacientes pode ter apontado mais de uma categoria.

** Algumas falas selecionadas de cada categoria

Quando indagados sobre o seguimento às orientações, encontramos que seis pacientes dos 10 que receberam orientações, referiram não seguir as orientações fornecidas. Os motivos pelos quais atribuíram tal feito, como demonstrado na Tabela 3 , relacionaram-se a três categorias: "incompatibilidade com sua realidade" (o paciente considera que os cuidados a serem tomados não se encaixam em seu cotidiano), "atribuição a locus externo de controle" (nesse caso foram incluídos aqueles pacientes que acreditavam que o problema da "não adesão" estava relacionado a fatores alheios ao próprio indivíduo) e "não adesão por indisposição à mudança". Este resultado, por sua vez, não se assemelha aos achados anteriores ${ }^{(6)}$ pelos quais, dos 19 (19/29) pacientes com valvopatia mitral que receberam informação, $17(89,5 \%)$ responderam que seguiam as orientações.

Tabela 3 - Distribuição da freqüência e da porcentagem das respostas dos pacientes $(n=06)$ que referiram não seguir as orientações fornecidas, segundo as categorias temáticas, Campinas, 1999

\begin{tabular}{|c|c|c|}
\hline CATEGCRIAS TEMÚTICOS & $\mathrm{NP}^{\mathrm{P}}$ & of \\
\hline Incompatibilidade com sua realidade & 1 & 167 \\
\hline Atribuição a kxvsextemo de controle & 3 & 500 \\
\hline Não adesão por indisposiç̃o à mudança & 2 & 33,3 \\
\hline
\end{tabular}

O problema da adesão ou da falta de adesão tem sido muito discutido nas últimas três décadas, sendo ressaltado os benefícios e por conseguinte a importância da adesão aos tratamentos medicamentosos e não medicamentosos implicados nas doenças de caráter crônico. Fatores como idade, sexo, raça, nível instrucional, condições para o autocuidado têm sido relacionados como intervenientes na adesão ${ }^{(12)}$. Dessa forma, cresce nas últimas duas décadas a preocupação com a adoção de intervenções, que através da educação e saúde possam efetivamente auxiliar o paciente na mudança e manutenção dos comportamentos relacionados à adesão. Assim, parece valer para os pacientes valvopatas as mesmas recomendações feitas pela Liga Mundial de Hipertensão em 1994, sobre a educação do paciente hipertenso, ou seja, é importante que sejam adotadas estratégias que consigam influenciar 0 comportamento do paciente induzindo-o a mudança e manutenção, guiando-o em direção a crenças, valores, percepções e atitudes em relação à doença, que lhe permitam ajustar-se bem à nova condição de vida determinada pela doença ${ }^{(13)}$.

Mais uma vez destacamos que é importante buscar conhecer a história que está por trás da crença que o paciente explicita, ou seja, o que ele realmente quer dizer através de um dado enunciado. E para isto, não existem regras, uma vez que o significado é tão variado, quanto é única a subjetividade de cada um. É este conhecimento, porém, que vai permitir o estabelecimento de intervenções educativas, ou ainda de promoção de saúde que sejam peculiares a cada indivíduo, e portanto, mais efetivo.

Isto posto, fica clara a necessidade de conhecermos, a partir da ótica do paciente, o que ele precisa e tem vontade de aprender ${ }^{(14)}$. Ao avaliarmos 0 desejo da população estudada em receber outras orientações sobre a doença e/ou tratamento, verificamos que apenas um paciente referiu preferir não recebê-la, pelo fato de no momento não estar sentindo nenhuma sintomatologia. Dentre os 11 pacientes interessados em receber novas orientações, constatamos maior freqüência em duas categorias: "adição de elementos para construir melhor sua representação da doença e/ou do seu estado de saúde" 
e "como a doença influirá na sua vida", assim como nos mostra a Tabela 4.

Tabela 4 - Distribuição da freqüência e da porcentagem das respostas dos pacientes $(n=11)$ que especificaram quais as orientações que gostariam de receber sobre sua doença e/ou tratamento, segundo as categorias temáticas, Campinas, 1999

\begin{tabular}{|c|c|c|}
\hline CATEGCRIASTEMÁTICAS & $\mathrm{NP}^{*}$ & $\sigma_{f} \neq$ \\
\hline $\begin{array}{l}\text { Adição de elementos para construir melhor sua } \\
\text { representação da doença ejou do seu estado de saúde }\end{array}$ & 4 & 36,4 \\
\hline $\begin{array}{l}\text { Como a doença influirá na sua vidainecessidade de } \\
\text { mudança de estilo de vida }\end{array}$ & 6 & 54,5 \\
\hline Relação da doença com a necessidade de intervenção & 2 & 18,2 \\
\hline
\end{tabular}

* Cada um dos pacientes pode ter apontado mais de uma categoria

A representação que o paciente tem sobre sua doença, por sua vez, pode estar relacionada à imagem corporal, um conceito que resulta da noção que a pessoa tem de si mesma ${ }^{(15)}$. A imagem corporal está associada a vivências afetivas em relação às partes do corpo, e cada um a elabora de modo absolutamente individual, acentuando ou modificando as relações existentes entre as diferentes partes, em função dos mecanismos de personalidade e de toda sua vivência passada e presente ${ }^{(16)}$. Assim, é importante, num programa educativo, que se busque além de identificar as crenças que o sujeito emite, atrelá-las à sua história de vida.

Desta forma, parece-nos claro que, um programa junto a este grupo de indivíduos, deva prever um conjunto de atividades que auxilie o paciente a obter os elementos dos quais necessita para construir melhor a representação que têm sobre sua doença, bem como dados que o permitam estabelecer um novo caminho a ser percorrido, considerando-se a perspectiva de vivência crônica com a doença. Assim, os educadores de saúde necessitam de destreza e sensibilidade para ajustar suas intervenções, de modo a atender às expectativas do paciente dentro do que se possa garantir 0 direcionamento para a adoção de comportamentos saudáveis ${ }^{(14)}$.

Quando indagados se realizavam alguma atividade física regularmente, pouco mais da metade (7/12) dos pacientes referiu não fazê-lo. Destes sete pacientes, cinco referiram ter alguma limitação física para atividade física aeróbica (ou da vida cotidiana). Destes cinco, apenas dois referiram ser a limitação percebida, 0 motivo para a não realização de atividade física regular. Os outros três (3/5), associados aos dois pacientes (2/7) que referiram não ter limitação, alegaram que o(s) motivo(s) que os levavam a não fazer a atividade física era( $\mathrm{m})$ o fato de não gostar de fazê-la e/ou não achála necessária. Dos cinco pacientes (5/12) que referiram fazer atividade física regular, quatro (4/5) relataram apresentar algum grau de limitação relacionado proporcionalmente à intensidade da atividade.

Portanto, dos dados obtidos, podem ser apreendidos dois pontos críticos na abordagem destes pacientes em relação ao exercício físico. 0 primeiro deles refere-se à limitação percebida por estes indivíduos para a atividade; o outro, refere-se ao "não gostar" de fazê-lo (porque não acredita na sua importância, qualificando-a como desnecessária, ou ainda como fonte de desprazer).

Em relação à limitação percebida, vale destacar que nesse grupo de pacientes ela pode ser realmente física e/ou estar vinculada à representação que o paciente tem sobre seu processo de adoecimento. Como já visto, o conjunto de sintomas apresentados pelo paciente valvopata é crítico, a ponto de ser um dos indicadores da necessidade da intervenção cirúrgica ${ }^{(17)}$, porém a forma como 0 indivíduo percebe estes sintomas contribui para sua magnitude e implicações na vida cotidiana.

Algumas considerações sobre 0 adoecer do coração sob uma visão psicanalítica são relatas em estudo prévio ${ }^{(18)}$, onde o autor destaca o enunciado psicanalítico que data de $1900^{(19)}$, o qual afirma que a mente é uma "eflorescência" do corpo, e que toda atividade psíquica deriva, em última instância, da estimulação somática. Problemas graves, no entanto, surgem quando os afetos são radicalmente expelidos da mente e lançados para o corpo, e a expressão psicossomática substitui a capacidade de sentir. "Nesses casos o sintoma físico ocupa o lugar da dor psíquica. Teremos, então, um indivíduo psiquicamente anestesiado ou desinformado sobre 0 universo de seus afetos e doente fisicamente" ${ }^{\text {"(18) }}$. Assim, a limitação do indivíduo para exercer atividade física pode não estar na esfera física, mas emocional. A compreensão deste fato é crucial para a adoção de intervenções adequadas, para que não sejam tomadas condutas que desconsiderem a esfera psíquica do indivíduo. É portanto fundamental mudar a expressão: "o problema é real ou psíquico" para "o problema real é físico ou psíquico". Pois, quando o problema é emocional", nem sempre é percebido o quão profunda pode ser a origem desse problema. "Não é que o paciente tenha escolhido sentir no corpo, ao invés de admitir que a dor é psíquica; não é que prefira remédios a psicoterapia; não é que tenha medo de desbravar o inconsciente, como às vezes pensamos; é possível que Ihe seja realmente impossível discriminar o que está se passando com ele naquele momento. Cobrar-lhe que entenda que o que sente é emocional; é como esperar que qualquer um de nós compreenda instantaneamente uma língua que nunca aprendeu"(18). É importante, assim, compreender a origem do sentimento de limitação que 0 paciente refere.

Por outro lado, há que se considerar a presença da sintomatologia. Sendo assim, a adoção de condutas que avaliem adequadamente o "status" físico do paciente e sua capacidade funcional e a orientação adequada podem auxiliar o indivíduo a confiar mais em sua capacidade física, e com isso, minimizar sua percepção de limitação.

Uma vez que a maioria dos pacientes valvopatas possui sintomas decorrentes da insuficiência cardíaca congestiva - ICC, observa-se que dados recentes ${ }^{(20)}$ demonstraram que pacientes com 
ICC, classificação funcional I-III, desde que estáveis, podem obter benefícios substanciais a partir de programas de condicionamento, nos quais são submetidos a sessões freqüentes e regulares de atividade aeróbica, por determinado período de tempo, sem maiores efeitos colaterais. É destacado ainda que 0 treino dos pacientes é harmonioso e capacita a uma melhora considerável na força muscular em todos os níveis, capacitando os pacientes a ganhar em autonomia e na qualidade de vida ${ }^{(21)}$. Melhoras, mesmo que modestas, em sua capacidade de realizar exercício podem melhorar significativamente sua capacidade para tarefas diárias ${ }^{(20)}$.

Ao levarmos em conta os benefícios que o exercício regular, uma vez bem indicado, pode trazer a estes pacientes, é fundamental que sejam desenvolvidas intervenções que auxiliem o indivíduo a conhecer efetivamente sua capacidade física para tal e, principalmente, a descobrir o prazer que pode existir na realização da atividade física.

Considerando-se que para a manutenção da qualidade de vida seja importante 0 aspecto nutricional do indivíduo, levantamos alguns dados sobre a alimentação deste grupo de pacientes. Para avaliarmos o grupo estudado em relação à ingestão de uma dieta equilibrada, utilizamos a classificação dos alimentos proposta em estudo prévio ${ }^{(22)}$. Observou-se, que todos os entrevistados possuíam pelo menos as duas principais refeições do dia (almoço e jantar), sendo que a maioria (8/12) apresentava as duas principais refeições do dia equilibradas.

No que se refere à alteração no estilo de vida em decorrência da presença da valvopatia, encontramos no grupo estudado oito pacientes (8/12), que referiram modificação em suas vidas em decorrência da doença. As categorias encontradas foram "modificação da potência de ação" e "mudança na afetividade", com freqüência de $62,5 \%$ e 50\%, respectivamente. Estes resultados são similares aos achados do estudo com portadores de valvopatia mitral ${ }^{(6)}$, em que verificou-se que do total de 29 pacientes portadores de insuficiência e estenose mitral entrevistados, 23 referiram modificação em suas vidas, em decorrência da valvopatia, destacando as mesmas categorias, cujas freqüências foram de 69,6 e $47,8 \%$, respectivamente.

Com relação à categoria "mudança na afetividade", observou-se em um estudo comparativo com o objetivo de avaliar as reações emocionais e as alterações em nível de imagem corporal de auto-estima e autoconceito em pacientes coronariopatas e valvopatas que aguardavam tratamento cirúrgico, que a maior parte dos pacientes apresenta, em seu relato, dificuldade para conviver com as alterações que a doença causou em sua rotina de vida, sentindo-se ameaçados pelas restrições a que possam vir a se submeter no pós-operatório e após a alta hospitalar ${ }^{(15)}$.

Assim, o enfermeiro precisa, primeiro, reconhecer e avaliar o conhecimento singular que o indivíduo e família trazem consigo sobre a experiência da doença em suas vidas; e segundo, atuar como profissional instruído e criativo na ajuda ao paciente/família no planejamento e adaptação de sua conduta em relação à doença ${ }^{(5)}$. A percepção do contexto em que indivíduos conduzem suas vidas e a identificação de modos criativos de ajuste às mudanças associadas com a valvopatia em suas vidas, são elementos cruciais para 0 estabelecimento de intervenções efetivas de enfermagem.

Ao avaliarmos os dados referentes à modificação na vida após o tratamento médico, verificamos, como mostra a Tabela 5 , que dos oito pacientes que referiram modificações em suas vidas, a maioria $(87,5 \%)$ citou aspectos relacionados à categoria "modificações relacionadas à sintomatologia". Dentre os quatro (4/12) pacientes que referiram não haver nenhuma modificação em suas vidas após o tratamento médico, os motivos citados para tal relato incluem-se nas seguintes categorias: "inalteração nas atividades de trabalho", "inalteração na sintomatologia" e "tratamento recente".

Tabela 5 - Distribuição da freqüência e da porcentagem dos pacientes $(n=08)$ que referiram modificações percebidas em sua vida após 0 início do tratamento médico, segundo as categorias temáticas, Campinas, 1999

\begin{tabular}{|c|c|c|}
\hline CATEGCRIASTEMÁTICAS & $N P \neq$ & $\sigma_{0} \neq$ \\
\hline Modificações relacionadas à sintomatologia & 7 & 87,5 \\
\hline Modificações relacionadas ao hábito alimentar & 1 & 125 \\
\hline Modificaçōes relacionadas à auto-confiança & 1 & 125 \\
\hline
\end{tabular}

* Cada um dos pacientes pode ter apontado mais de uma categoria

No presente estudo, de todos os pacientes entrevistados, quatro (4/12) referiram não realizar atividade sexual; dentre estes, apenas um (1/4) referiu a não realização devido ao problema de saúde. Além disso, dos oito (8/12) pacientes que afirmaram realizar atividade sexual, sete (7/8) referiram não possuir limitação para tal e, destes, seis (6/7) relataram satisfação com a mesma. Resultado bastante diferente foi encontrado no estudo com portadores de valvopatia mitral ${ }^{(6)}$, no qual a maioria dos pacientes $(73,9 \%)$ com vida sexual ativa apresentava limitações no intercurso das relações, que foram associadas à sintomatologia decorrente da doença. As autoras observaram ainda que o cansaço e a dispnéia foram os sintomas mais citados por aqueles pacientes. Além disso, ao investigarem se o grupo estudado considerava sua atividade sexual satisfatória, constatou-se que, mesmo com a presença da sintomatologia acarretada pela valvopatia, foi pequena a parcela de pacientes que relatou insatisfação na atividade sexual. A insatisfação, por sua vez, foi relacionada à doença ou atribuída à indisposição para vida sexual plena.

Existem dificuldades para o estudo da atividade sexual, visto que há vários fatores psicológicos, fisiológicos e éticos a serem considerados, tanto do paciente/parceiro, quanto do profissional. A começar pelos aspectos fisiológicos, temos que a atividade sexual 
não pode ser estudada meramente como atividade física. Os sujeitos estão geralmente em uma posição corporal (deitado); utilizam grupos musculares diferentes daqueles usados enquanto caminhamos ou andamos de bicicleta; não estão em um estado metabólico constante (especialmente durante o breve período de orgasmo) e podem estar fazendo uma combinação de trabalhos isotônicos e isométricos, 0 que dificulta a quantificação dos efeitos fisiológicos do sexo. Os aspectos emocionais e culturais ligados à sexualidade, por sua vez, são responsáveis, em muitas situações, pela criação de barreiras para a discussão do assunto entre o paciente e o profissional. Outro ponto que há de se considerar também é o fato da sexualidade do sujeito estar profundamente ligada à sua história de vida, de modo que constitui um equívoco avaliar isoladamente os problemas a ela relacionados, e não dentro de um contexto específico do indivíduo ${ }^{(23)}$.

\section{CONSIDERAÇÕES FINAIS}

Os pacientes estudados caracterizaram-se pelo predomínio do sexo masculino, idade média de 49 anos ( $\pm 16,5$ anos), baixo nível de escolaridade, provenientes de Campinas e região e com exercício de alguma atividade profissional. Quanto ao diagnóstico médico, a maioria possuía dupla lesão da valva aórtica ou insuficiência aórtica isolada. Mais da metade dos pacientes não sabia referir a causa de seu problema, sendo que vários atribuíram a doença a fatores não relacionados. Foi constatado que os pacientes possuem

\section{REFERÊNCIAS BIBLIOGRÁFICAS}

1. Guimarães GCQCP, Viana LAC. Características do paciente valvopata com diagnóstico de excesso de líquido e déficit de conhecimento sobre a doença. Rev Soc Cardiol Estado de São Paulo 1993; 3 (4 Suppl A):5-8.

2. Blumental JÁ, Mark DB. Quality of life recovery after cardiac surgery. [editorial comment]. Psychosomatic Medicine 1994; 56:213-5.

3. Silva MAD. A importância da manutenção da qualidade de vida. Rev Soc Cardiol Estado de São Paulo 1996; 6(5):657-60.

4. Romano BW. Qualidade de vida: teoria e prática. Rev Soc Cardiol Estado de São Paulo 1993; 3 (6):6-9.

5. King KM. Valve disorder: theorial and practical nursing perspectives. CJCN 1994; 5(1):13-7.

6. Kubo KM, Colombo RCR, Gallani MCBJ, Noronha R. Subsídios para assistência de enfermagem a pacientes com valvopatia mitral. Rev Latino-am Enfermagem 2001 maio; 9(3):33-42.

7. Patton MQ. Enhancing the quality and credibility of qualitative analysis. Health Serv Res 1999; 34(5 Pt 2):1190-208. representações sobre sua doença que vão desde a concepção (simples) da fisiopatologia, à sua repercussão na vida do sujeito, estabeleciam relação com sua etiologia, bem como com a necessidade de intervenção terapêutica. Alguns pacientes, entretanto, demonstraram construir com dificuldade sua representação, parecendo ser evidente a falta de elementos para fazê-lo, reforçado pelo desejo de receber mais orientações. A doença levou a modificações na vida da maioria dos sujeitos entrevistados, sendo observado o relato de modificação da potência de ação e da afetividade. Mais da metade dos pacientes, entretanto, relatou algum grau de melhora da qualidade de vida após instituição do tratamento médico. A maioria dos sujeitos entrevistados não relatou queixas quanto à sexualidade. Quanto à alimentação, observou-se que todos os pacientes não realizavam desjejum equilibrado, enquanto que mais da metade relatava almoço e jantar equilibrados. Pouco mais da metade (7/12) dos pacientes referiu não realizar nenhum tipo de atividade física regularmente, sendo que pela maioria dos entrevistados foi relatado algum grau de limitação para realização de atividade física tipo aeróbica e/ou atividades de vida diárias.

Os dados coletados são consistentes no sentido de subsidiar a elaboração de um programa educativo junto aos pacientes valvopatas, destacando-se que os mesmos permitiram a detecção dos problemas apresentados pelo grupo estudado, cuja análise apontou caminhos para conhecer-se a subjetividade dos individuos estudados. Subjetividade esta que deve sempre ser um dos elementos norteadores do planejamento de enfermagem que visa educação em saúde.

8. Prefeitura Municipal de Campinas. Secretaria de Planejamento. Sumário de Dados. n.1. População: região de Campinas, 1993.

9. Resnekov L. Aortic valve stenosis: management in children and adults. Postgrad Med 1993; 93(6):107-22.

10. Braunwald E. Tratado de medicina cardiovascular. $4^{a}$ ed. São Paulo (SP): Roca; 1996.

11. Eisenberg MJ. Rheumatic heart disease in the developing world: prevalence, prevention and control. Eur Heart J 1993; (14): 122-8.

12. Sarquis LMM, Dell'Acqua MCQ, Gallani MCBJ, Moreira RM, Bocchi SCM, Tase TH, et al. A adesão ao tratamento na hipertensão arterial: análise da produção científica. Rev Esc Enfermagem USP 1998; 32 (4): 335-53.

13. Strasser T, Grueninger UJ, editors. Educating the hypertensive patient: a manual for practising physicians and nurses. Geneva: Hypertension League (WHL); 1994.

14. Hansen M, Streff MM. Patient education: practical guidelines. In: Pollock ML, Schmidt DH, editors. Heart disease and rehabilitation. $3^{\mathrm{a}}$ ed. USA: Human Kinetics; 1995. p. 277-85. 
15. Pires CA, Sharovsky LL, Romano BW. Coronariopatas e valvopatas: impacto emocional da cirurgia cardíaca. Estudo comparativo. Rev Soc Cardiol Estado de São Paulo 1994; 4 (5 Suppl A):1-7.

16.Van Kolck OL. O desenho da figura humana. In: Van Kolck OL. Testes projetivos gráficos no diagnóstico psicológico São Paulo (SP): EPU; 1984. p.13-54.

17. Carabello BA, Crawford FA. Valvular heart disease. N Engl J Med 1997; 337(1):32-41.

18. Svartman B. Adoecer do coração: visão psicanalítica. Rev Soc Cardiol Estado de São Paulo1994; 4 (1 Suppl A):5-8.

19. Freud S. La interpretación de los sueños. In: Freud S. Obras Completas. Madri: Biblioteca Nueva; 1948.
20. European Heart Failure Training Group. Experience from controled trials of physical training in chronic heart failure. Protocol and patient factors in effectiveness in the improvement in exercise tolerance. Eur Heart J 1998; (19): 466-75.

21. Koch M, Dovard G, Broustet JP. The benefit of graded physical exercise in chronic heart failure. Chest 1992; 101(5): 231-5.

22. Boog MCF, Motta DG, Bon AMX. Alimentação natural: prós e contras. São Paulo (SP): Ibrasa; 1985.

23. Skinner JS. Sexual relations. In: Pollock ML, Schmidt DH, editors. Heart disease and rehabilitation. $3^{\mathrm{a}}$ ed. USA: Human Kinetics; 1995. p. $367-78$ 